\title{
The impact of interfaces in laminated packaging on transport of carboxylic acids
} \author{
A. Viridén ${ }^{\mathrm{b}, \mathrm{f}}, \mathrm{M}$. Nicholas ${ }^{\mathrm{b}, \mathrm{f}}, \mathrm{N}$. Lorén ${ }^{\mathrm{b}, \mathrm{g}}, \mathrm{A}$. Larsson ${ }^{\mathrm{a}, \mathrm{b}}$ \\ a Department of Chemistry and Chemical Engineering, Chalmers University of Technology, Gothenburg, Sweden \\ b SuMo Biomaterials, VINN Excellence Centre, Chalmers University of Technology, Gothenburg, Sweden \\ ${ }^{\mathrm{c}}$ Department of Mathematical Sciences, Chalmers University of Technology, Sweden \\ d Tetra Pak AB, New Material Design, Lund, Sweden \\ e Department of Chemistry "Ugo Schiff" and CSGI, University of Florence, Italy \\ ${ }^{\mathrm{f}}$ Astra Zeneca, RED Gothenburg, Sweden \\ g Soft Materials Science, SP Food and Bioscience, Gothenburg, Sweden
}

S. Gårdebjer ${ }^{\mathrm{a}, \mathrm{b}, *}$, T. Gebäck $^{\mathrm{b}, \mathrm{c}}, \mathrm{T}$. Andersson ${ }^{\mathrm{b}, \mathrm{d}}$, E. Fratini $^{\mathrm{e}}$, P. Baglioni $^{\mathrm{e}}$, R. Bordes $^{\mathrm{a}, \mathrm{b}}$,

\section{A R T I C L E I N F O}

\section{Article history:}

Received 6 April 2016

Received in revised form

7 June 2016

Accepted 30 June 2016

Available online 5 July 2016

Keywords:

Permeability

Laminates

Interfaces

Polymers

Packaging

\begin{abstract}
A B S T R A C T
The permeability of oleic and acetic acid through low density polyethylene (LDPE) and ethylene acrylic acid (EAA) have been measured using diffusion cells. In addition, the permeability through combinations of LDPE and EAA in the form of laminates with different numbers of layers has been determined. Oleic acid shows an almost 30 times higher permeability compared to acetic acid, which was partly explained by the adsorption of oleic acid to the film surface during the permeability experiment. In addition, the permeability is lower for both oleic and acetic acid in the laminates compared to the pure films. The decreased permeability can be explained by the presence of crystalline domains close to the interface. This is supported by SAXS data which suggests an ordering of polymer chains in the EAA film close to the interface. In summary, the results show that it is possible to create barrier materials with decreased permeability, which is interesting for example in the packaging industry.
\end{abstract}

(c) 2016 Elsevier B.V. All rights reserved.

\section{Introduction}

Food packaging materials function as barriers and aim to preserve and protect a food product from the surrounding environment [1]. Glass and metal have historically been used as barriers, and during the 20th century, plastics became popular to use as packaging materials, and a vast range of plastics with difference properties has been developed to fulfill the various needs encountered. For example, low-density polyethylene (LDPE), which is a generically used plastic in packaging materials, is a good moisture barrier but a poor oxygen barrier [2]. Therefore, a plastic barrier is often combined with other materials i.e. other plastics or foil, often via co-extrusion, to create a laminate and an improved barrier. The natural result of the formation of a laminate is the creation of additional interfaces between the layers. The width and shape of the interface often vary and depend strongly on the polymers involved [3]. In addition, the interface properties often

\footnotetext{
* Corresponding author at: Department of Chemistry and Chemical Engineering, Chalmers University of Technology, Gothenburg, Sweden.

E-mail address: sofie.gardebjer@chalmers.se (S. Gårdebjer).
}

differ from those of the bulk, which can affect the final barrier properties [4]. For example, molecular weight and polydispersity of a polymer have been shown to have impact on the interface, since high polydispersity and low molecular weight could result in the formation of a broader interface [5]. It has also been shown that shorter chains tend to assemble at the interface, hence lowering the interfacial tension [6] as a result of advantageous entropy gained by the system by excluding the longer chains from the interface [5]. The assembly of shorter polymer chains at the interface has also been shown to be responsible for the presence of a more ordered structural arrangement close to the interface, which is usually called transcrystalline layer [7-9]. A transcrystalline layer can also form between two immiscible polymers as a result of high polydispersity and the diffusion of shorter chains at the interface [9]. In that case, a decreased permeability for the laminate is expected due to the increased crystallinity at the interface.

Mass transport - or permeability - is directly connected to solubility and diffusivity of the permeant. However, the nature of the polymeric material (cross-linking, crystallinity, and substituents), possible addition of plasticizers, compatibilizers, stabilizers or fillers and temperature are all important factors 


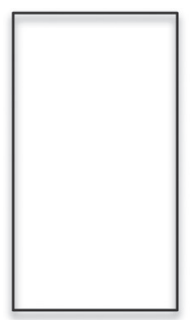

A

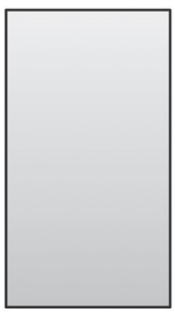

B

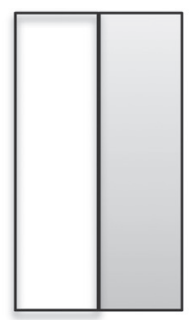

A B

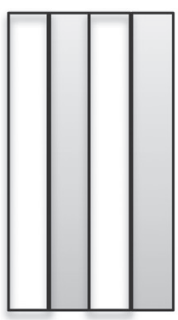

A B A B

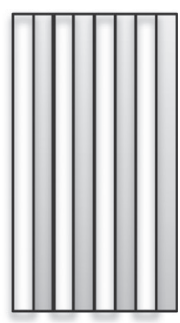

A B A BA B A B

Fig. 1. Schematic representation of the pure films and the laminates, $A=$ low density polyethylene (LDPE), $B=e$ ethylene acrylic acid (EAA).

influencing the permeability [10]. Crystalline parts in a polymeric material are often considered to be impermeable for small molecules. Hence, the mass transport of a permeant occurs mainly in the amorphous regions [11]. The mass transport can also depend on the free volume within the polymer and the segmental mobility of the polymer chains. Even though laminates are widely used in the packaging industry, only few studies are focused on the permeability of a liquid and what impact the interfaces may have on the total transport. There are many studies focusing on the permeability of gases through polymeric film materials, however [12$16]$.

In this study, we investigated the impact of the film layering on both the mass transport and the total permeability. Our hypothesis was that the presence of additional interfaces could drastically reduce the mass transport and total permeability of a permeant. To test this hypothesis, the permeability of oleic and acetic acid was measured for two pure polymer films; low density polyethylene (LDPE) and ethylene acrylic acid (EAA) as well as combinations of them consisting of up to eight layers (Fig. 1) while keeping the total film thickness constant. The structure of the film materials was investigated by small angle X-ray scattering (SAXS) and wide angle X-ray diffraction (WAXD), and the thickness of the interface was estimated using time-of-flight secondary ion mass spectrometry (TOF-SIMS). These results were further used to calculate an interfacial permeability and compare it to experimental values.

\subsection{Theoretical basis}

\subsubsection{Diffusion through a polymeric film}

For a barrier film without defects the primary mechanism for mass transport of a permeant from one side of a film to the other is diffusion, driven by the concentration gradient created in the experimental setup. Molecular diffusion in one dimension can be described by Fick's first law:

$J=D \frac{d c}{d x}$ where $J$ is the flux, $D$ is the diffusion coefficient of the permeant and $d c / d x$ its concentration gradient. The diffusion coefficient is assumed to be independent of position and time. Furthermore, when it is assumed that steady state has been reached, i.e. when the concentration within the film does not vary with time, Eq. (1) can be rewritten into Eq. (2):

$J=\frac{D A}{h}\left(c_{1}-c_{2}\right)$

where $A$ is the area exposed to the permeant, $h$ is the total film thickness, $c_{1}$ and $c_{2}$ are the concentrations at the surfaces of the films in Fig. 2a. In the case where the permeant is dissolved in aqueous solution surrounding the film barrier and thus not accumulated at the surface by an adsorption process, Eq. (2) presumes that the aqueous boundary layers on both sides of the film do not significantly affect the total transport process. Therefore, $c_{1}$ and $c_{2}$ can be related to the concentrations in the chambers by considering the partition coefficient, $K$, i.e. the ratio between the concentration in the chamber and at the surface of the film (Fig. 2a).

$K=\frac{c_{1}}{c_{d}}=\frac{c_{2}}{c_{a}}$

where $c_{d}$ and $c_{a}$ are the concentrations in the donor and acceptor regions respectively. Furthermore, Eqs. (2) and (3) can be rewritten into Eq. (4):

$J_{i}=D_{i} K_{i} A \frac{\left(c_{d}-c_{a}\right)}{h}=P_{i} A \frac{\left(c_{d}-c_{a}\right)}{h}$

The diffusion coefficient $\left(D_{i}\right)$ times the partition coefficient $\left(K_{i}\right)$ is equal to the permeability $\left(P_{i}\right)$ for permeant $i$ according to Eq. (4).

Fig. 2a shows the case when the partition coefficient $K$ is equal to 1 , i.e. the concentration of the interior film surface is the same as the concentration outside the film. However, for solid materials this is not always true, and Fig. 2b shows the situation when $K \ll 1$, i.e. the concentration within the film is lower than outside the

(a)

(b)

(c)
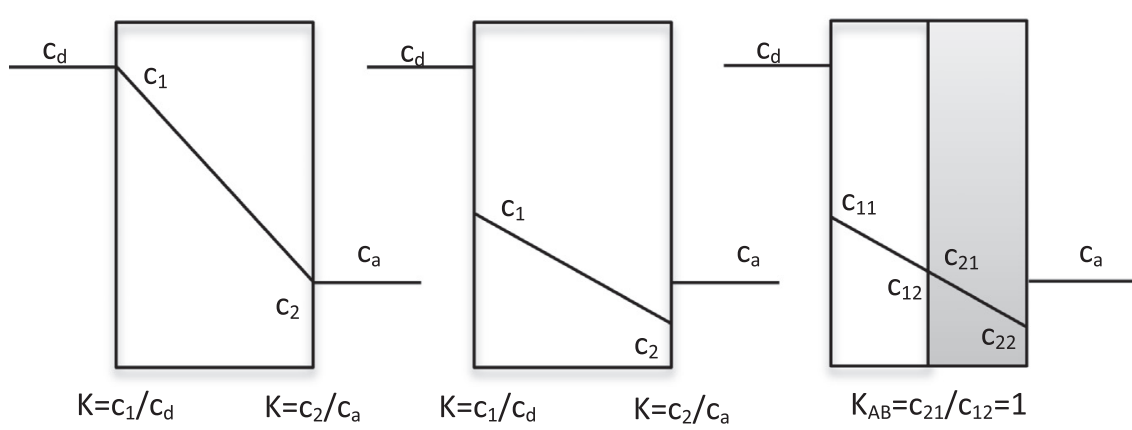

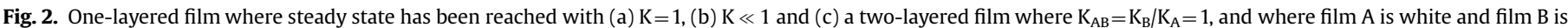
gray. Donor and acceptor chamber are on the left and right side, respectively. 
film. Fig. $2 \mathrm{c}$ refers to the same scenario for the case of a membrane constituted by two different layers of different materials where the interface does not affect the total permeability, i.e. $K=1$ for the interface.

\subsubsection{Diffusion through layered structures}

For many applications in the industry, laminates are common, e.g. packaging materials, diapers and wound dressings. If the mass transport $d m / d t$ is constant through each layer, a pseudo steady state is reached, and then Eq. (5) can be used to calculate the total permeability of a laminate according to the Ideal Laminate Theory [17]:

$\frac{\mathrm{h}_{\text {tot }}}{\mathrm{P}_{\text {tot }}}=\sum_{1}^{\mathrm{n}} \frac{\mathrm{h}_{\mathrm{i}}}{\mathrm{P}_{\mathrm{i}}}$

where $P_{i}$ and $h_{i}$ are the permeability and the thickness of each layer, respectively. This equation assumes that the mass transport is the same regardless of the order of the individual layers $[17,18]$. In addition, the equation is only valid for defect-free films. Fig. $2 \mathrm{c}$ shows an example for which Eq. (5) is valid, with the expressions $K_{A B}$ (and $K_{B A}$ ) being the partition coefficients in the interface formed between materials $A$ and $B$. For a film $A$ with the partition coefficient $K_{A}$ and a film B with partition coefficient $K_{B}$, this equation is valid only in the case when $K_{A B}=1 / K_{B A}=K_{B} / K_{A}$, meaning that there is no difference of permeability regardless of how many layers the material consists of. This equation also assumes that the presence of the interface does not affect the total mass transport through the material. It is however possible to add an extra term to Eq. (5) [19] in order to account for a possible effect of the interfaces in the total transport:

$\frac{\mathrm{h}_{\text {tot }}}{\mathrm{P}_{\text {tot }}}=\sum_{1}^{\mathrm{n}} \frac{\mathrm{h}_{\mathrm{i}}}{\mathrm{P}_{\mathrm{i}}}+\sum_{1}^{n} \frac{h_{\text {int }}}{P_{\text {int }}}$

where $h_{\text {int }}$ and $P_{\text {int }}$ are the thickness and permeability of the interfaces in the film. This additional sum includes all the interfaces present in a laminate, but the condition of $K_{A B}=1 / K_{B A}=K_{B} / K_{A}$ is still valid.

\section{Experimental}

The A and B materials in this study were low-density polyethylene (LDPE) and the copolymer ethylene acrylic acid (EAA) in a $1: 1$ composition for all films. The film materials were produced in pilot scale at Tetra Pak, Lund, Sweden via hot-melt extrusion. The films consisted of either the pure materials LDPE or EAA or different layered combinations as shown in Fig. 1. Regardless of the number of layers, the total thickness of all films was kept constant at $100 \pm 2 \mu \mathrm{m}$. To guarantee homogeneity of the film materials, samples were taken at different areas of the films. The cross-section was also studied by a microscope (Olympus BH2 polarizing microscope) prior to measurements.

\subsection{Size exclusion chromatography (SEC-RI)}

Size exclusion chromatography refractive index (SEC-RI) was used to determine the average molar mass and polydispersity for the pure films as well as for the 2-layered film. The films were dissolved in 1,2,4-trichlorobenzene (TCB) at $135{ }^{\circ} \mathrm{C}$ overnight at a concentration of $1 \mathrm{mg} / \mathrm{ml}$. The solution was filtered through a metal filter with a pore size of $0.45 \mu \mathrm{m}$ before injection into SEC equipment (Waters Alliance GPCV2000, USA). TCB was used as eluent, and universal calibration with polystyrene was performed. Number and weight averaged molecular weights $\left(\mathrm{M}_{\mathrm{n}}\right.$ and $\left.\mathrm{M}_{\mathrm{w}}\right)$ and polydispersity index $\left(\mathrm{M}_{\mathrm{w}} / \mathrm{M}_{\mathrm{n}}\right)$ were calculated accordingly.
2.2. Small angle $X$-ray scattering/wide angle $X$-ray diffraction (SAXS/ WAXD)

SAXS/WAXD measurements were carried out with a HECUS S3MICRO camera (Kratky-type) equipped with a position-sensitive detector (OED $50 \mathrm{M}$ ) containing 1024 channels of width $54 \mu \mathrm{m}$. Cu $K \alpha$ radiation of wavelength $\lambda=1.542 \AA$ was provided by an ultrabrilliant point micro-focus X-ray source (GENIX-Fox 3D, Xenocs, Grenoble), operating at a maximum power of $50 \mathrm{~W}(50 \mathrm{kV}$ and $1 \mathrm{~mA}$ ). The sample-to-detector distance was $281 \mathrm{~mm}$. The volume between the sample and the detector was kept under vacuum (1 mbar) during the measurements to minimize scattering from the air. The Kratky camera was calibrated in the small angle region using silver behenate $(\mathrm{d}=58.34 \AA$ ) [20] while lupolen $(\mathrm{d}=4.12 \AA)$ was used as a reference for the wide-angle region. SAXS curves were obtained in the scattering vector, $q$, range between 0.01 and $0.54 \AA^{-1}$, assuming that $q=(4 \pi \mid \lambda) \sin \theta$, and $2 \theta$ is the scattering angle. The WAXD region covered in the experiment was from 1.3 to $1.9 \AA^{-1}$. Films were placed into an open demountable cell. The experiment was conducted in transmission geometry with the beam perpendicular to the film surface; the total thickness of the sample used was $200 \mu \mathrm{m}$ in all cases (i.e. two parallel films). The temperature was set to $25^{\circ} \mathrm{C}$ and controlled by a Peltier element, with an accuracy of $0.1^{\circ} \mathrm{C}$. All scattering curves were corrected for the empty cell contribution considering the relative transmission factor.

\subsection{Time-of-flight secondary ion mass spectrometry (TOF-SIMS)}

The chemical composition of the 8-layered film was studied with TOF-SIMS (Model TOF-SIMS 5, ION-TOF GmbH, Germany) equipped with $\mathrm{a} \mathrm{Bi}_{3}{ }^{+}$analysis beam and an argon-cluster sputter beam. The argon cluster beam had argon clusters of a size centered at 1500 argon atoms per cluster. An acceleration of $10 \mathrm{keV}$ was used. A $200 \times 200 \mu \mathrm{m}^{2}$ crater was sputtered, and a $50 \times 50 \mu \mathrm{m}^{2}$ area in the center of the crater was analyzed using the $30 \mathrm{keV} \mathrm{Bi}_{3}{ }^{+}$ analysis beam. $32 \times 32$ pixels with 1 shot per pixel were analyzed using a sputter time of $5 \mathrm{~s}$ followed by a pause of $0.5 \mathrm{~s}$. This sequence was used to follow the concentration changes with depth.

\subsection{Permeability measurements}

Permeability measurements were performed in home-made polypropylene diffusion cells, as earlier described in the literature [21]. In short, the diffusion cells consist of sealed donor and acceptor chambers, where a film is placed in a $5 \mathrm{~cm}$ hole between the chambers. The thickness of the films was recorded at five points using a micrometer (Mitoyo $10 \mathrm{C}-112 \mathrm{MB}$, Japan) prior to the permeability measurements. $40 \mathrm{ml}$ of MilliQ-water was added to each chamber, and $10 \mu \mathrm{l} \mathrm{of}{ }^{3}[\mathrm{H}]$-oleic acid or ${ }^{3}[\mathrm{H}]$-acetic acid was added to the donor chamber (maximum final concentration in donor chamber is $2.5 \cdot 10^{-4} \mathrm{mg} / \mathrm{ml}$ ). The solution was stirred by placing the diffusion chambers on a rotating table revolving at $50 \mathrm{rpm}$ (Edmund Bühler 7400, Germany). The permeation was followed by taking out aliquots of $500 \mu \mathrm{l}$ from the acceptor chamber and immediately adding the same amount of pure MilliQ-water. Samples were assayed with scintillation liquid, Ultima Gold, and run in a scintillation analyzer (Tri-Carb B2810TR, PerkinElmer, USA). Permeability of the films was calculated according to Eq. (4).

\subsection{Quartz crystals microbalance (QCM)}

The oscillation frequencies of pure silica covered QCM crystals (Q-Sense Sensor QSX $303 \mathrm{SiO}_{2}$ ) was measured in a QCM-D instrument (model E4, Q-Sense Göteborg, Sweden) in air. 
Subsequently, the crystals were spin-coated with a thin layer of LDPE or EAA from a pre-heated solution consisting of $0.5 \mathrm{wt} \%$ of the polymers dissolved in $p$-xylene. The spin-coater used was from Laurell Technologies Corporation, USA (Model WS-650MZ-23NPP). The QCM crystals were heated to $85^{\circ} \mathrm{C}$ for $1 \mathrm{~min}$ prior to spincoating. $100 \mu \mathrm{l}$ of the polymer solution was added to the crystal, and the speed was set to $800 \mathrm{rpm}$ for $30 \mathrm{~s}$, followed by $2000 \mathrm{rpm}$ for $60 \mathrm{~s}$. The spin-coated crystals were left at ambient conditions several days prior to QCM measurement. The oscillation frequencies for the spin-coated crystals was measured in order to calculate an approximate thickness of the spin-coated polymer layer.

The adsorption of oleic and acetic acid was measured at $20^{\circ} \mathrm{C}$ with a baseline corresponding to $5 \mathrm{wt} \%$ aqueous ethanol solution for oleic acid and MilliQ-water for acetic acid. The amount of oleic acid required to perform QCM measurements was not soluble in water, therefore a $5 \mathrm{wt} \%$ ethanol solution was used. Five different solutions of oleic and acetic acid were injected from $1.2 \cdot 10^{-5} \mathrm{mg} /$ $\mathrm{ml}$ to $1.2 \cdot 10^{-1} \mathrm{mg} / \mathrm{ml}$ by reducing the dilution of acids in steps of ten, where the concentration used in the permeability experiment is between the second and third concentration. The mass absorbed to the film surfaces was calculated.

\section{Results and discussion}

According to the suggested hypothesis, the presence of interfaces in layered film materials is believed to contribute to a decreased permeability. To study the hypothesis, the physical properties of the materials and the interfaces were characterized by SEC-RI, SAXS/WAXD and TOF-SIMS. Permeability of oleic and acetic acid was measured in diffusion cells over several weeks for the laminates, and the results were compared to conventional models. Additionally, the adsorption to the surface of the two carboxylic acids was studied using QCM.

\subsection{Properties of the interface}

Fig. 3 shows the SEC-RI chromatogram for the LDPE, EAA and 2-layered films where both $M_{w}$ and $M_{n}$ were higher for the LDPE film compared to the EAA film. The average $M_{w}$ for LDPE was $1.8 \cdot 10^{5} \mathrm{~g} / \mathrm{mol}$ while EAA had an average $\mathrm{M}_{\mathrm{w}}$ of $7.8 \cdot 10^{4} \mathrm{~g} / \mathrm{mol}$. The average $M_{n}$ for LDPE was $1.3 \cdot 10^{4} \mathrm{~g} / \mathrm{mol}$ while EAA had a $M_{n}$ of $2.7 \cdot 10^{3} \mathrm{~g} / \mathrm{mol}$. The polydispersity was 26 for EAA compared to

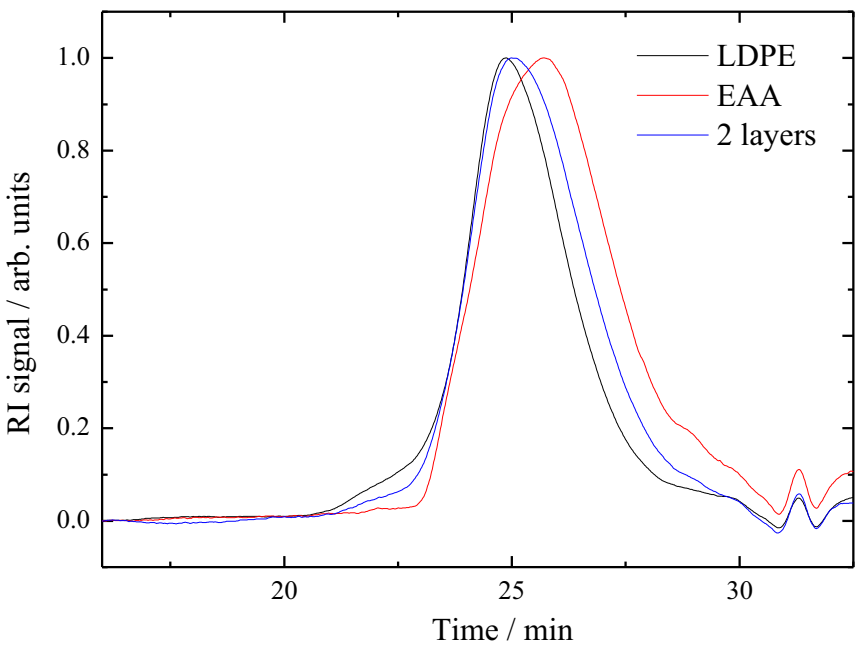

Fig. 3. SEC-RI chromatogram for LDPE (black), EAA (red) and the 2-layered film (blue). (For interpretation of the references to color in this figure legend, the reader is referred to the web version of this article.)
LDPE that had a polydispersity of 13 . The high polydispersity of EAA could result in a denser material close to the interface $[9,22]$ since the shorter chains can more easily diffuse and lower the interfacial tension between the two polymers [5,6]. This will have implications on the formation of crystalline domains at the interface.

A simultaneous SAXS/WAXD investigation was conducted on the two pure films as well as on the 2-layered samples to gather a detailed picture of the film nanostructure. Fig. 4a shows the SAXS patterns characteristic of the investigated films. The broad peak in the SAXS region indicates a correlation length that in the case of these polymeric films corresponds to the repetition of amorphous chains sandwiched between crystalline lamellae as schematically illustrated in the inset of Fig. 4a. Fig. 4b shows the WAXD patterns obtained on the same films. All the samples show two broad Bragg peaks as a result of the crystalline ordering at the molecular level (i.e. the characteristic inter-chain distances). SAXS and WAXD peaks were modeled using a Gaussian function. This is a simple approach to extract the peak position and width. In the WAXD case only the most intense peak at about $1.5 \AA^{-1}$ was considered. The parameters obtained from the fitting are reported in Table 1 along with the average distance in the real space associated to the peaks as extracted by $2 \pi / q_{\text {peak }}$.

As is evident from the data reported in Table 1, the amorphouscrystalline correlation length $(\xi)$ equals $16.2 \mathrm{~nm}$ for LDPE and $12.0 \mathrm{~nm}$ for EAA for the pure films. In the case of the 2-layered LDPE/EAA sample a value of $14.6 \mathrm{~nm}$ was measured instead of 13.8 as expected for a non-interacting layered system. (Expected values are shown in parenthesis in Table 1 and calculated according to $\xi=2 \pi / q_{\max }$ sAXs). The width of the peak is also reported as a measure of the polydispersity of the amorphous-crystalline domain size which follows the order LDPE $>$ LDPE/EAA $>$ EAA. Similar evidence could be extracted from the WAXD patterns where the chains in the case of LDPE film are more packed (i.e. the average inter-chain distances are smaller) than the EAA case. Considering the width of the peaks in the WAXD case, it is possible to speculate that the LDPE sample presents ordered domains that are more extended than in the EAA case. Again the LDPE/EAA case is closer to LDPE than expected. Hence, the main finding of the SAXS/WAXD investigation is that the co-extrusion of the two polymers forces the coextruded EAA to generate a structure arrangement closer to the LDPE case at the interface (i.e. with a correlation length about $1 \mathrm{~nm}$ more than expected, and more packed). McEvoy and coworkers have shown that transcrystalline layers thinner than $5 \mu \mathrm{m}$ can form between LDPE and EAA with 3\% of acrylic acid [9], which could explain the results obtained here.

The data from TOF-SIMS experiment are shown in Fig. 5, where the intensity variation with sputter time is shown for negative ions of mass $16\left(\mathrm{O}^{-}\right)$and $17\left(\mathrm{OH}^{-}\right)$respectively. The molecular weights chosen should only be present in the EAA film and not in the LDPE film. As can be seen in the figure, the intensity for $\mathrm{O}^{-}$in the EAA films goes from 6000 to zero and for OH from 4200 to zero when the sputtering by argon source reaches the LDPE film. The time it takes to sputter through the interface can be used to calculate an approximate thickness of the interface. It should be noted that the topography of the interface can be important for the time required to go from the EAA to the LDPE film, meaning that a rough interface can result in longer times than for a planar interface. However, the time required is not believed to be due to an uneven sputtering because depth resolution has been shown to be in the range of $4-5 \mathrm{~nm}$ using argon clusters as a sputtering source [23]. For the 8-layered film the interfacial thickness for each interface was calculated to be in the range of $2-2.5 \mu \mathrm{m}$. Since there are eight interfaces in total, the total thickness of interfaces in that film should be maximum $17.5 \mu \mathrm{m}$. It has earlier been shown that there is some miscibility between LDPE and EAA when both polymers 
(a)

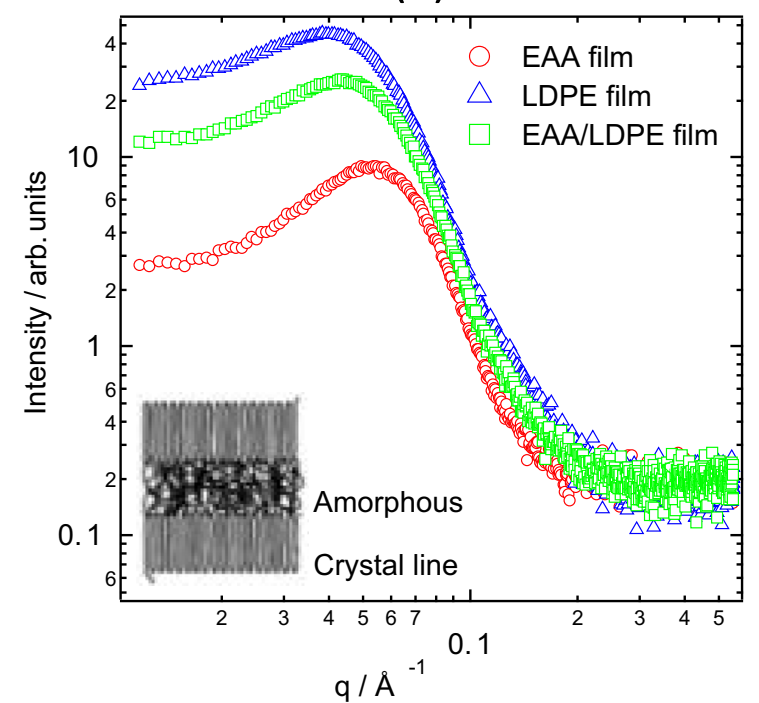

(b)

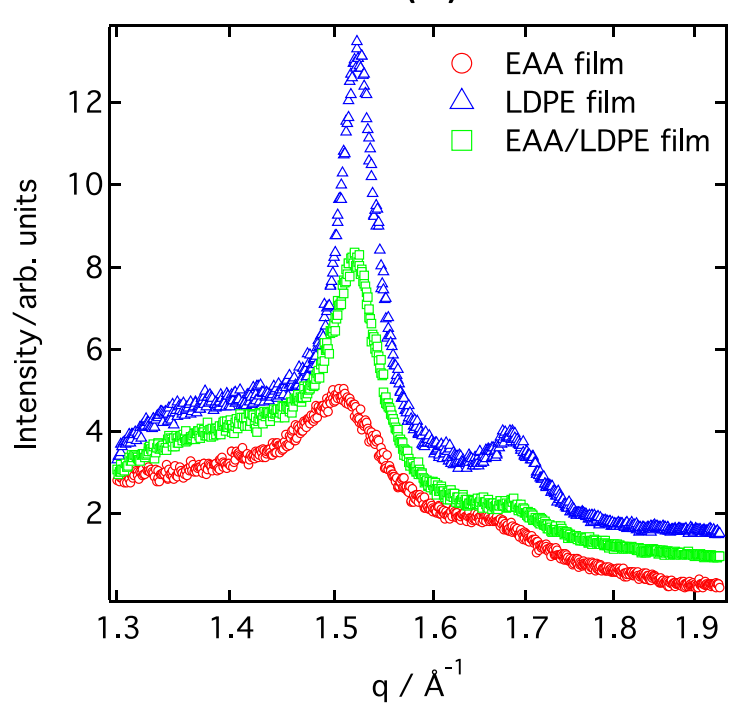

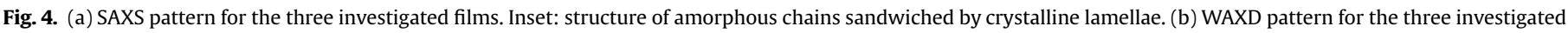
films. WAXD patterns were arbitrarily shifted along the y-axis for the sake of clarity.

Table 1

Nanoscale order parameters (SAXS) and crystalline parameters (WAXD) obtained from the analysis of Fig. 4. A simple Gaussian has been used to extract the parameters from the broad peaks. Numbers in parenthesis show the theoretical mean values expected for the bulk polymers in LDPE/EAA.

\begin{tabular}{|c|c|c|c|}
\hline & LDPE & EAA & LDPE/EAA \\
\hline \multicolumn{4}{|l|}{ SAXS } \\
\hline$q_{\max }, \operatorname{saxs}\left(\AA^{-1}\right)$ & 0.0387 & 0.0525 & $0.0428(0.0456)$ \\
\hline$\sigma_{\text {SAXS }}($ sigma, Å) & 0.027 & 0.025 & $0.026(0.026)$ \\
\hline$\xi(\mathbf{n m})$ & 16.2 & 12.0 & $14.6(13.8)$ \\
\hline \multicolumn{4}{|l|}{ WAXD } \\
\hline $\mathbf{q}_{\max , \text { WAXD }}\left(\AA^{-1}\right)$ & 1.522 & 1.501 & $1.518(1.512)$ \\
\hline$\sigma_{\text {WAXD }}($ sigma, $\AA$ ) & 0.025 & 0.056 & $0.030(0.041)$ \\
\hline D $_{\text {interchain }}(\AA)$ & 4.12 & 4.18 & $4.14(4.15)$ \\
\hline
\end{tabular}

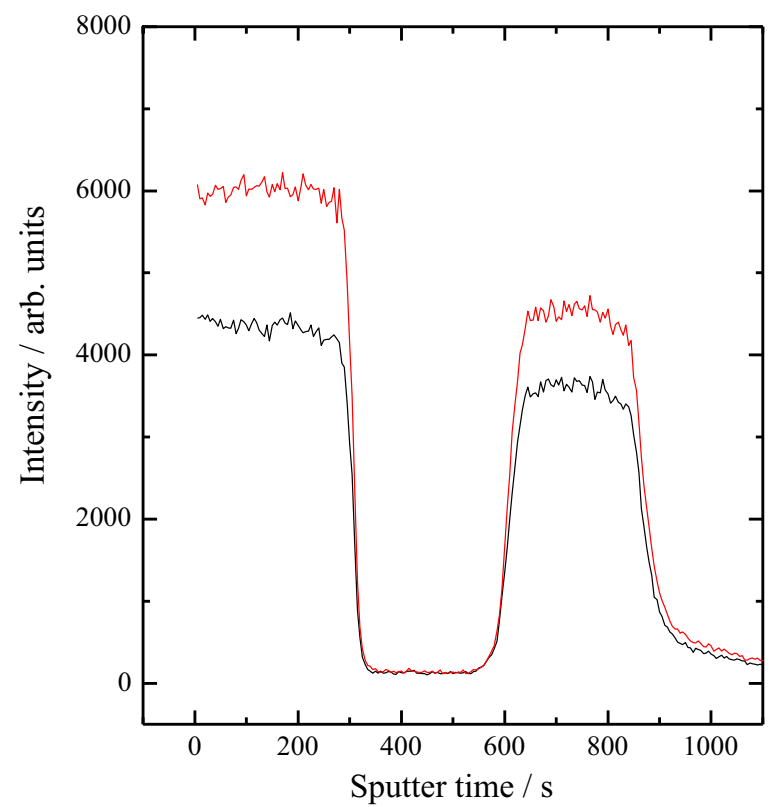

Fig. 5. TOF-SIMS data for an 8-layered film with the EAA material placed closest to the sputter beam, the black line represents $\mathrm{O}^{-}$and the red $\mathrm{OH}^{-}$. The interfacial thickness is approximated from the sputter time. (For interpretation of the references to color in this figure legend, the reader is referred to the web version of this article.) are in the molten state [9], which is in agreement with an approximate thickness of $2-2.5 \mu \mathrm{m}$ for an interface. In addition, the high polydispersity of both polymers also support a thicker interface [5].

\subsection{Permeability and adsorption measurements}

Fig. 6 shows the experimental permeability (gray bars) of oleic and acetic acid for the pure LDPE and EAA films as well as for 2-, 4and 8-layered films. The theoretical permeability for the laminates was calculated according to Eq. (5) and is shown as white bars in the same figure. Using the Ideal Laminate Theory and the conditions given in Eq. (5), the calculations were based on the experimental values for pure LDPE and pure EAA films, and it was assumed that the interface was very thin (interfacial thickness set to $\mathrm{nm}$ scale compared to bulk thickness of $12.5-50 \mu \mathrm{m}$ ). Furthermore, interference with the permeant was assumed to be similar in the interface as well as the bulk material and therefore negligible. The experimental data for oleic acid shows a substantial decrease when going from one layer to two layers (one interface) and the decrease continues slowly when going to eight layers (seven interfaces). In contrast, for acetic acid, the decrease in permeability going from one layer to two layers is small, but the decrease for four and eight layers is substantial. By comparing the experimental data with the theoretical values, it is clear that the presence of the interfaces has an impact on the total permeability for the laminates. According to the SAXS/WAXD findings, the difference in the nanostructure of the LDPE/EAA film and the denser chain packing imposed by the co-extrusion process could be responsible for the differences obtained in the permeability experiments.

The highest permeability was found for oleic acid in pure LDPE film with a value of $101 \pm 5 \cdot 10^{-15} \mathrm{~m}^{2} / \mathrm{s}$ while in the case of EAA it decreases to a value of $69 \pm 6 \cdot 10^{-15} \mathrm{~m}^{2} / \mathrm{s}$. The same trend is seen for acetic acid, where the LDPE has a permeability of $2.5 \pm 1 \cdot 10^{-15} \mathrm{~m}^{2} / \mathrm{s}$ and EAA $2.0 \pm 0 \cdot 10^{-15} \mathrm{~m}^{2} / \mathrm{s}$. Permeability, $\mathrm{P}$, is defined as $\mathrm{P}=\mathrm{DK}$ (see Eq. (4)), where D is the diffusion coefficient and $\mathrm{K}$ is the partition (or solubility) coefficient. Due to the chemical structure of the two carboxylic acids, it is likely that oleic acid will have a lower diffusion coefficient but a higher solubility in the two polymers than acetic acid. The increase of solubility is therefore dominating over the loss of diffusivity and this hypothesis could justify the higher permeability observed for oleic acid in 
(a)

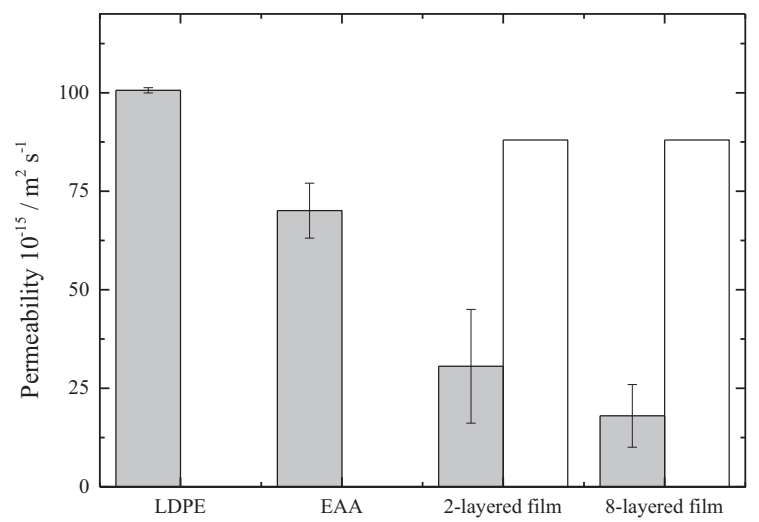

(b)

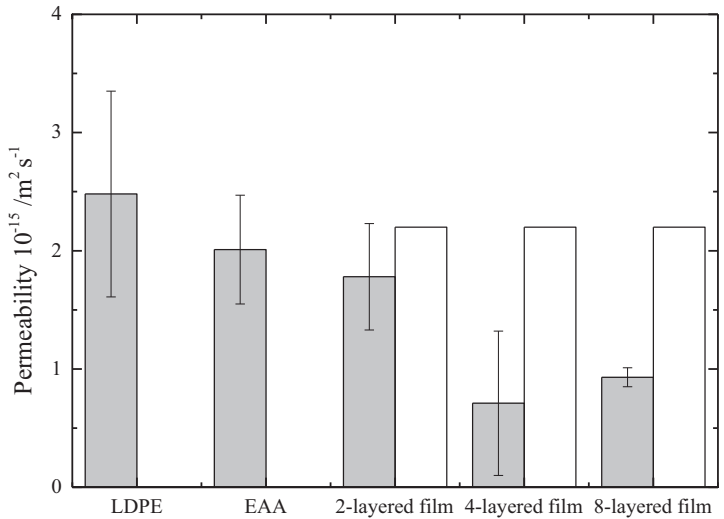

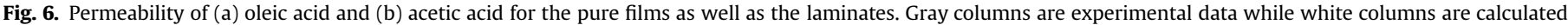
values based on the assumption that the interfaces do not influence the permeability, see Eq. (5). Note that the scales on the y-axis are different.

both LDPE and EAA. A possible explanation for the decreased permeability of both oleic and acetic acid in EAA compared to LDPE is that the acrylic acid groups in the EAA material can interact with the head group of the acids, resulting in hindered diffusion and hence lower total permeability. It is remarkable that the permeability is about 30 times higher for oleic acid compared to acetic acid for the two pure film materials. The large difference in the permeability for oleic and acetic acid might be explained by a tendency of the former to adsorb to hydrophobic surfaces [24]. This was tested using QCM and the results are presented in Section 3.2.1.

\subsubsection{Adsorption measurements}

To test if an increased adsorption was the explanation for the higher permeability of oleic acid, QCM measurements were performed on spin-coated films of LDPE and EAA. The thickness of the spin-coated LDPE films onto the QCM crystals was calculated according to Eq. (7):
$\Delta m=-\frac{\Delta f \cdot \mathrm{C}}{n}$

where $\Delta m$ is the absorbed mass, $\Delta f$ is the variation in resonance frequency for the uncoated and LDPE-coated crystal, $n$ is the overtone numbers and $\mathrm{c}$ is a constant characteristic of the equipment used which is determined to $17.7 \mathrm{ng} / \mathrm{cm}^{2}$. Overtone number three, five and seven were used to calculate the thickness of the spin-coated LDPE layers to be approximately $100 \pm 20 \mathrm{~nm}$.

Increasing concentrations of the acids were flowed over the spin-coated LDPE and EAA films, and the mass absorbed was calculated according to Eq. (7) (Fig. 7a and b), assuming adsorption of a rigid film. To quantify and achieve a larger span of concentrations for the acids, a baseline of $5 \mathrm{wt} \%$ of ethanol in water was used for oleic acid, and pure water was run for acetic acid. Fig. 7a shows the mass of oleic acid adsorbed onto the spin-coated LDPE and EEA films with increasingly concentrated oleic acid added over time. The arrows show when solution of increasing concentrations of oleic acid are injected in the measurement cell. (a)

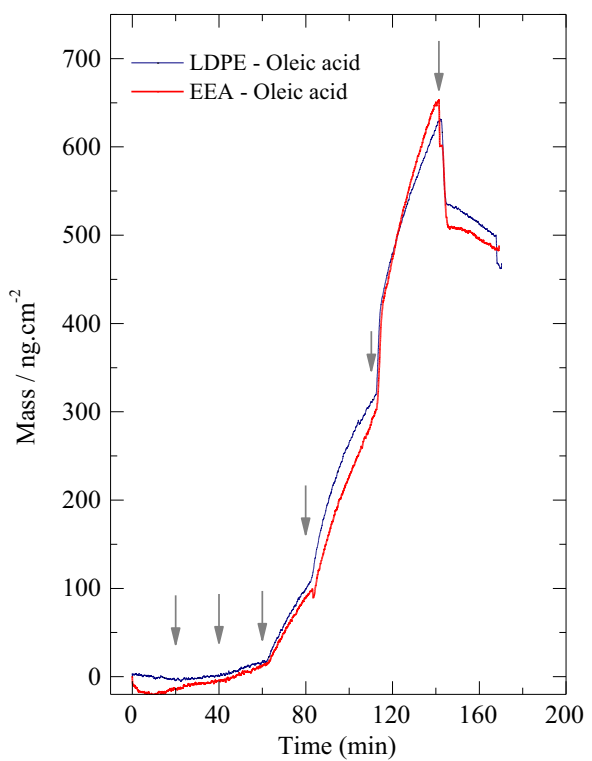

(b)

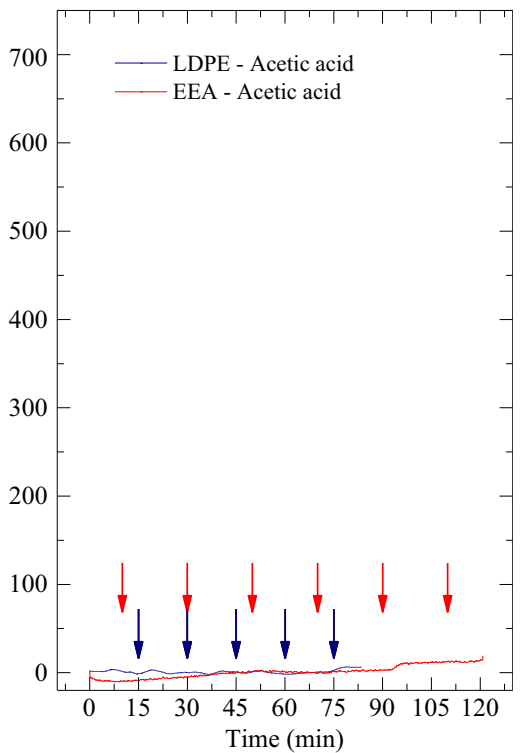

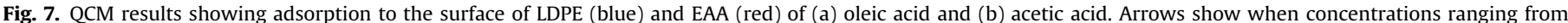

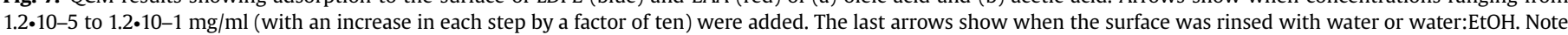

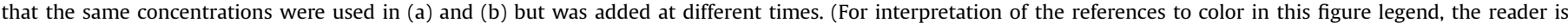
referred to the web version of this article.) 
Table 2

Calculated values of the additional term hint/Pint in Eq. (6), as well as the calculated ratio of permeability of the interfaces.

\begin{tabular}{llll}
\hline Material & $\mathrm{h}_{\text {int }} / \mathrm{P}_{\text {int }}$ (oleic acid) & $\mathrm{h}_{\text {int }} / \mathrm{P}_{\text {int }}$ (acetic acid) & Ratio $\mathrm{P}_{\text {int ac }} / \mathrm{P}_{\text {int ol }}$ \\
\hline 2-layered film & $2.1 \cdot 10^{9}$ & $1.2 \cdot 10^{10}$ & 6 \\
8-layered film & $4.6 \cdot 10^{9}$ & $7.0 \cdot 10^{10}$ & 15 \\
\hline
\end{tabular}

\section{Table 3}

Suggested interfacial thicknesses as well as calculated interfacial permeability according to Eq. (6).

\begin{tabular}{llll}
\hline Material & $\begin{array}{l}\text { Interfacial thickness } \\
(\mu \mathrm{m})\end{array}$ & $\begin{array}{l}\mathrm{P}_{\text {int }} \text { (oleic acid) } \\
\left(\mathrm{m}^{2} / \mathrm{s}\right)\end{array}$ & $\begin{array}{l}\mathrm{P}_{\text {int }} \text { (acetic acid) } \\
\left(\mathrm{m}^{2} / \mathrm{s}\right)\end{array}$ \\
\hline 2-layered film & 2.5 & $1.2 \cdot 10^{-15}$ & $0.2 \cdot 10^{-15}$ \\
4-layered film & 7.5 & $\mathrm{~N} . \mathrm{A}$. & $0.1 \cdot 10^{-16}$ \\
8-layered film & 17.5 & $3.8 \cdot 10^{-15}$ & $0.2 \cdot 10^{-15}$ \\
\hline
\end{tabular}

Already when the second concentration of oleic acid was added, there seems to be some adsorption based on the increased mass observed. When the third concentration is added there is clearly an increasing mass upon the polymer films. Adsorption of oleic acid occurred to the surface of both LDPE and EAA, even at concentrations lower compared to what is used in the permeability experiment. Furthermore, in the final step of the run with oleic acid, the $5 \mathrm{wt} \%$ of ethanol water solution was injected to wash away non-adsorbed oleic acid, but the adsorption of oleic acid is almost irreversible. These observations are in agreement with previous report on adsorption of oleic acid at various surfaces [24]. Fig. 7b shows the results from acetic acid, where no adsorption onto the surface was observed, even when higher concentrations compared to what is used in the permeability experiment were tested. The preferential adsorption of oleic acid to the surface may be part of the explanation for the 30 times higher permeability registered for oleic acid as compared to acetic acid as shown in Fig. 6a and b.

\subsubsection{Permeability for laminates}

As stated earlier, the permeability of laminates can be decomposed in a finite sum of terms accounting for the interfacial domains and the bulk region, respectively. It is therefore possible to calculate the additional term $h_{\text {int }} / P_{\text {int }}$ for the laminates. The values were estimated using Eq. (6) based on the experimental data from the permeability experiments, and associated results are shown in Table 2. The approximate thickness of the interface as determined by TOF-SIMS experiments was subtracted from A and B layers. The ratio $P_{\text {int ac }} / P_{\text {int ol }}$ was calculated, and as shown in Table 2 , the ratio is almost three times higher for the 8-layered film compared to the 2-layered film. This large difference between the ratios suggests that the permeability of oleic acid is lower through the interface compared to acetic acid. The permeability - according to Eq. (4) depends on the thickness of the film, the diffusion coefficient of the permeant and the partition coefficient for the film. Since the thickness is the same for all films, the permeability will depend on $\mathrm{D}$ and $\mathrm{K}$. The lower permeability of oleic acid may then be explained by the different chain lengths of acetic and oleic acid, where the longer oleic acid may have a higher partition coefficient but should have a slower diffusion compared to acetic acid.

Assuming that the interfacial thickness obtained from TOFSIMS is similar in the 2- and 8-layered films, a theoretical value of the interfacial permeability can be calculated. The thicknesses of the interfaces are shown in Table 3, and the interfacial permeability is calculated according to Eq. (6). The calculated interfacial permeability is lower compared to the experimental values for the films presented in Fig. 6a and b, where LDPE had an experimental permeability of $101 \cdot 10^{-15} \mathrm{~m}^{2} / \mathrm{s}$ for oleic acid and $2.5 \cdot 10^{-15} \mathrm{~m}^{2} / \mathrm{s}$ for acetic acid, which indeed is much higher than the calculated interfacial permeability for both oleic and acetic acid in the laminates. This discrepancy can be explained as a result of the nonhomogenous ordering of polymer chains in the vicinity of the interface. Such ordering was observed using SAXS/WAXD, which gave an average value over the whole film. Microscopic structural changes that result in local variation of permeability are not reflected in the measurement. This means that there may be parts that are less permeable while other parts close to the interface are as permeable as the bulk material. If so, the total experimental permeability is expected to decrease, as observed. Hence, it seems important to take the presence of interfaces in a film material into consideration when producing packaging materials with barrier properties.

\section{Conclusions}

Our results have shown that it is possible to reduce the total permeability of oleic and acetic acid for a laminate compared to a pure film material. The decreased permeability was explained by ordering of polymer chains close to the interface formed between the two materials. SAXS/WAXD data showed that there was an ordering of the chains of the copolymer EAA close to the interface, probably caused by the co-extrusion of the two materials. The presence of crystals will hinder the transport of the acids through the film material, resulting in a decreased permeability. Even though the permeability decreased for the laminates, the permeability of oleic acid was almost 30 times higher compared to acetic acid. We used QCM to show that oleic acid adsorbs to the hydrophobic surfaces of both LDPE and EAA, while acetic acid do not. This adsorption was believed to result in a higher permeability for oleic acid compared to acetic acid. The results obtained in this study open up for possibilities to produce improved barrier materials using laminates instead of pure polymer films.

\section{Acknowledgments}

The VINN Excellence Center Sumo Biomaterials is gratefully acknowledged for financial support. Anders Mårtensson, Chalmers University of Technology, is acknowledged for help with SEC measurements. Consorzio Interuniversitario per lo Sviluppo dei Sistemi a Grand Interfase Center for Colloid and Surface Science (CSGI) and Ministreo dell'Istruzione, dell'Università e della Ricera (MiUR) are acknowledged for partial financial support.

\section{References}

[1] A. Ahmed, N. Ahmed, A. Salman, Critical issues in packaged food business, Br. Food J. 107 (2005) 760-780.

[2] C. Vasile, M. Pascu, Practical Guide to Polyethylene, Rapra Technology Limited, Shawbury, Shrewsbury, UK, 2005.

[3] B.K. Fink, R.L. McCullough, Interphase research issues, Comp. Part A: Appl. Sci. Manuf. 30 (1999) 1-2.

[4] S. Mukhopadhyay, B.L. Deopura, R. Alagiruswamy, Interface behavior in polypropylene, Composites J. Thermoplast. Comp. Mater. 16 (2003) 479-495.

[5] D. Broseta, et al., Molecular weight and poly-dispersity effects at polymerpolymer interfaces, Maromolecules 23 (1990) 132-139.

[6] S.H. Anastasiadis, I. Gancarz, J.T. Koberstein, Interfacial tension of immiscible polymer blends, temperature and molecular weight dependence, Macromolecules 21 (1988) 2980-2987.

[7] D.R. Fitchmun, S. Newman, Surface crystallization of polypropylene, J. Polym. Sci. Part A-2: Polym. Phys. 8 (1970) 1545-1564.

[8] N. Klein, et al., Determining the role of interfacial transcrystallinity in composite materials by dynamic mechanical thermal analysis, Composites 26 (1995) 707-712.

[9] R.L. McEvoy, S. Krause, Interfacial interactions between PE and PP and some 
ethylene-containing co-polymers, Macromolecules 29 (1996) 4258-4266.

[10] S.C. George, S. Thomas, Transport phenomena through polymeric systems, Prog. Polym. Sci. 26 (2001) 985-1017.

[11] D.H. Weinkauf, D.R. Paul, Effects of Structural Order on Barrier Properties, in Barrier Polymers and Structures, American Chemical Society, Dallas, Texas, 1990.

[12] C.E. Rogers, V. Stannett, M. Szwarc, Permeability Valves. Permeability of gases and vapors through composite membranes, Ind. Eng. Chem. 49 (1957) 1933-1936.

[13] R. Waack, et al., Permeability of polymer films to gases and vapors, Ind. Eng. Chem. 47 (1955) 2524-2527.

[14] J. Greener, et al., Moisture permeability through multilayered barrier films as applied to flexible OLED display, J. Appl. Polym. Sci. 106 (2007) 3534-3542.

[15] P.S. Holden, G.A.J. Orchard, I.M. Ward, A study of the gas barrier properties of highly oriented polyethylene, J. Polym. Sci. : Polym. Phys. Ed. 23 (1985) 709-731.

[16] P. Kofinas, R.E. Cohen, A.F. Halasa, Gas permeability of polyethylene/poly (ethylene-propylene) semicrystalline diblock copolymers, Polymer 35 (1994) 1229-1235.

[17] A. Grüniger, P. Rudolf von Rohr, Influence of defects in SiOx thin films in their barrier properties, Thin Solid Films 459 (2004) 308-312.

18] V. Stannett, Permeability of Plastic Films and Coated Paper to Gases and Vapors, Technical Association Of The Pulp And Paper Industry, New York, 2004.

[19] K.M. Furuheim, Synergy Effects between polyethylene and High Density Papers in Gas Barrier Materials - a morphological Study in NTNU Department of Chemical Engineering, Trondheim, Norway, 2002.

[20] T.N. Blanton, et al., Crystal structure determination of the silver carboxylate dimer $[\mathrm{Ag}(\mathrm{O} 2 \mathrm{C} 22 \mathrm{H} 43)] 2$, silver behenate, using powder X-ray diffraction methods, Powder Diffr. 26 (2011) 313-320.

[21] J. Hjärtstam, T. Hjertberg, Studies of the water permeability and mechanical properties of a film made of an ethyl cellulose-ethanol-water ternary mixture, J. Appl. Polym. Sci. 74 (1999) 2056-2062.

[22] T. Tränkner, M. Hedenqvist, U.W. Gedde, Molecular and lamellar structure of an extrusion-grade medium-density polyethylene for gas distribution, Polym. Eng. Sci. 34 (1994) 1581-1588.

[23] E. Niehuis, et al., Analysis of organic multilayers and 3D structures using $\mathrm{Ar}$ cluster ions, Surf. Interface Anal. 45 (2013) 158-162.

[24] E.R. Garland, et al., Structure of submonolayer oleic acid coverages on in organic aerosol particles: evidence of island formation, Phys. Chem. Chem. Phys. 10 (2008) 3156-3161. 